\title{
What is the optimal number of neoadjuvant chemotherapy cycles for resectable colorectal liver oligometastases?
}

\author{
Qichen Chen", Xingchen Li", Jianjun Zhao, Xinyu Bi, Zhiyu Li, Zhen Huang, Yefan Zhang, \\ Jianguo Zhou, Hong Zhao, Jianqiang Cai \\ Department of Hepatobiliary Surgery, National Cancer Center/National Clinical Research Center for Cancer/Cancer Hospital, Chinese Academy of \\ Medical Sciences and Peking Union Medical College, Beijing, China \\ Contributions: (I) Conception and design: H Zhao, J Zhou; (II) Administrative support: J Zhou, H Zhao, J Cai; (III) Provision of study materials or \\ patients: Q Chen, X Li; (IV) Collection and assembly of data: Q Chen, X Li, J Zhao; (V) Data analysis and interpretation: Q Chen, X Li, J Zhao, X \\ Bi; (VI) Manuscript writing: All authors; (VII) Final approval of manuscript: All authors. \\ \#These authors contributed equally to this work. \\ Correspondence to: Hong Zhao; Jianguo Zhou. Department of Hepatobiliary Surgery, National Cancer Center/National Clinical Research \\ Center for Cancer/Cancer Hospital, Chinese Academy of Medical Sciences and Peking Union Medical College, Beijing 100021, China. \\ Email: zhaohong@cicams.ac.cn; zjgty@hotmail.com
}

Background: The optimal number of neoadjuvant chemotherapy (NAC) cycles for resectable colorectal liver oligometastases (CLOM) remains unclear. The aim of this study was to investigate the optimal number of NAC cycles.

Methods: One hundred twenty-nine consecutive patients were included in this study. X-tile analysis was implemented to investigate the optimal cut-off point for NAC cycles. Propensity score matching was performed to reduce selection bias. Kaplan-Meier curves and Cox risk regression models were used to analyse progression-free survival (PFS) and overall survival (OS).

Results: The optimal cut-off point for NAC cycles was 5. There were no significant differences in R0 resection, pathological response or postoperative complications between the groups with a low number of NAC cycles group ( $\leq 5$ cycles, $n=80)$ and high number of NAC cycles $(>5$ cycles, $n=49)$. Patients with a high number of NAC cycles were more likely to have NAC toxicity than those with a low number of cycles $(87.8 \%$ vs. $65.0 \%, \mathrm{P}=0.004)$. Multivariate analysis revealed that $>5 \mathrm{NAC}$ cycles was an independent predictor of reduced PFS (HR $=1.808,95 \% \mathrm{CI}: 1.205-2.712, \mathrm{P}=0.004)$ and reduced $\mathrm{OS}(\mathrm{HR}=1.723,95 \%$ CI: 1.041-2.851, $\mathrm{P}=0.034)$. In the oxaliplatin-based regimen group, patients with a low number of NAC cycles had a better PFS ( $\mathrm{P}<0.001$, mPFS: 14.7 vs. 5.4 months) and better $\mathrm{OS}(\mathrm{P}=0.018$, mOS: 57.7 months $v s .41 .0$ months) than those with a high number of cycles. After 1:1 propensity matching (34 cases $v s .34$ cases), multivariate analysis revealed that $>5$ NAC cycles was an independent predictor of reduced PFS (HR $=2.265$, 95\% CI: 1.281-4.007, $\mathrm{P}=0.005)$ and reduced OS (HR =2.813, 95\% CI: 1.359-5.822, P=0.005). In the oxaliplatin-based regimen group, patients with a low number of NAC cycles had better $\mathrm{PFS}(\mathrm{P}<0.001$, mPFS: 17.5 vs. 5.6 months) and better OS ( $\mathrm{P}=0.008$, mOS: 59.0 vs. 31.8 months) than those with a high number of cycles.

Conclusions: Fewer than 5 NAC cycles was optimal for biologically resectable CLOM patients. Giving more than 5 NAC cycles was unnecessary because a higher number of NAC cycles has more unfavourable survival and higher NAC toxicities, while leading to similar R0 resection rates and pathological responses.

Keywords: Colorectal liver oligometastases (CLOM); neoadjuvant chemotherapy (NAC); cycles; outcomes

Submitted May 27, 2020. Accepted for publication Sep 30, 2020.

doi: 10.21037/atm-20-4289

View this article at: http://dx.doi.org/10.21037/atm-20-4289 


\section{Introduction}

Colorectal cancer is the fourth most common malignant tumour worldwide and the second leading cause of cancerrelated death (1). The liver is the most common metastatic organ for colorectal cancer. Liver metastasis is found in $15-25 \%$ of colorectal cancer patients upon diagnosis, while another $15-25 \%$ of patients have liver metastasis after primary colorectal cancer resection $(2,3)$. Liver resection is the most effectively curative treatment for colorectal cancer liver metastasis (CRLM) patients, with a 5 -year survival rate of $43 \%$ (4).

The 2016 ESMO guidelines divided metastatic colorectal cancer into two categories: oligometastatic disease and widespread systemic disease (5). Oligometastasis refers to an intermediate state in the process of tumour metastasis. It is a relatively early stage of biological invasion between localized primary tumours and widespread metastatic tumours. Oligometastatic disease confined to the liver is potentially curable. Aggressive local treatments, especially liver resection, may prolong the survival of patients with colorectal liver oligometastases (CLOM), leading to a 5-year overall survival (OS) rate of $45.9 \%(6,7)$. Neoadjuvant chemotherapy (NAC) can treat micrometastases, reduce the burden of tumours, and improve the rate of $\mathrm{R} 0$ resection (8). For biologically resectable CLOM patients, NAC is increasingly used as a potentially effective treatment strategy. However, it is still unclear how many cycles of NAC are needed to obtain the maximum benefits for biologically resectable CLOM. Some studies have shown that more than eight or more than nine cycles of NAC for CRLM patients did not add any benefit and led to the increased postoperative liver insufficiency and toxicities $(9,10)$. The National Comprehensive Cancer Network (NCCN) guidelines (11) recommend the option of NAC for resectable CRLM for a period of 2-3 months but do not specify how many cycles of NAC would benefit patients the most.

The optimal number of NAC cycles is vital for CLOM patients. Too many NAC cycles may lead to more NAC toxicities, increase the incidence of postoperative complications $(10,12)$ and potentially increase the risk of progression. An insufficient number of NAC cycles cannot achieve the maximum benefits from neoadjuvant therapy. To address this question, this study was conducted to investigate the optimal number of NAC cycles for patients with biologically resectable CLOM. We present the following article in accordance with the
STROBE reporting checklist (available at http://dx.doi. org/10.21037/atm-20-4289).

\section{Methods}

All participants gave informed consent before taking part in this study. This study was conducted with approval from the Institute Research Ethics Committee of the Cancer Hospital, Chinese Academy of Medical Sciences (ID: NCC2019C-016). The study conformed to the provisions of the Declaration of Helsinki (as revised in 2013).

\section{Patient population}

We retrospectively examined data from 407 CRLM patients who underwent primary liver resection at the Cancer Hospital, Chinese Academy of Medical Sciences, from December 2006 to May 2018. The final enrolled patients met the following inclusion criteria: (I) pathologically confirmed CRLM; (II) treatment with NAC; (III) colorectal liver oligometastases ( $\leq 5$ liver metastases and no preoperative extrahepatic metastases) $(5,7)$; and (IV) biological resectability (remaining liver volume more than $30-40 \%$ after liver resection). We excluded 278 patients based on the inclusion criteria: no prior NAC $(n=194)$; non-biologically resectable colorectal liver oligometastases $(\mathrm{n}=60)$; treatment with neoadjuvant radiotherapy $(\mathrm{n}=14)$ and lost to follow-up or incomplete clinical data $(\mathrm{n}=10)$. The final cohort consisted of 129 patients (Figure 1). Comorbidity was defined as chronic diseases (e.g., diabetes mellitus or pulmonary, cardiovascular, and other diseases).

\section{Treatment}

Patients received NAC with oxaliplatin- or irinotecanbased regimens, such as FOLFOX (5-fluorouracil/ leucovorin/oxaliplatin), XELOX (capecitabine/oxaliplatin) or FOLFIRI (5-fluorouracil/ leucovorin/irinotecan). The targeted therapy regimens combined with NAC included bevacizumab or cetuximab. The NAC regimens, number of NAC cycles and timing of surgery were determined by a multidisciplinary team (MDT) including surgeons, oncologists, pathologists and imaging physicians, as previously described $(13,14)$. NAC regiments were determined according to the characteristics of these regimens and the specific conditions of patients. When determining the number of NAC cycles for CRLM, the 


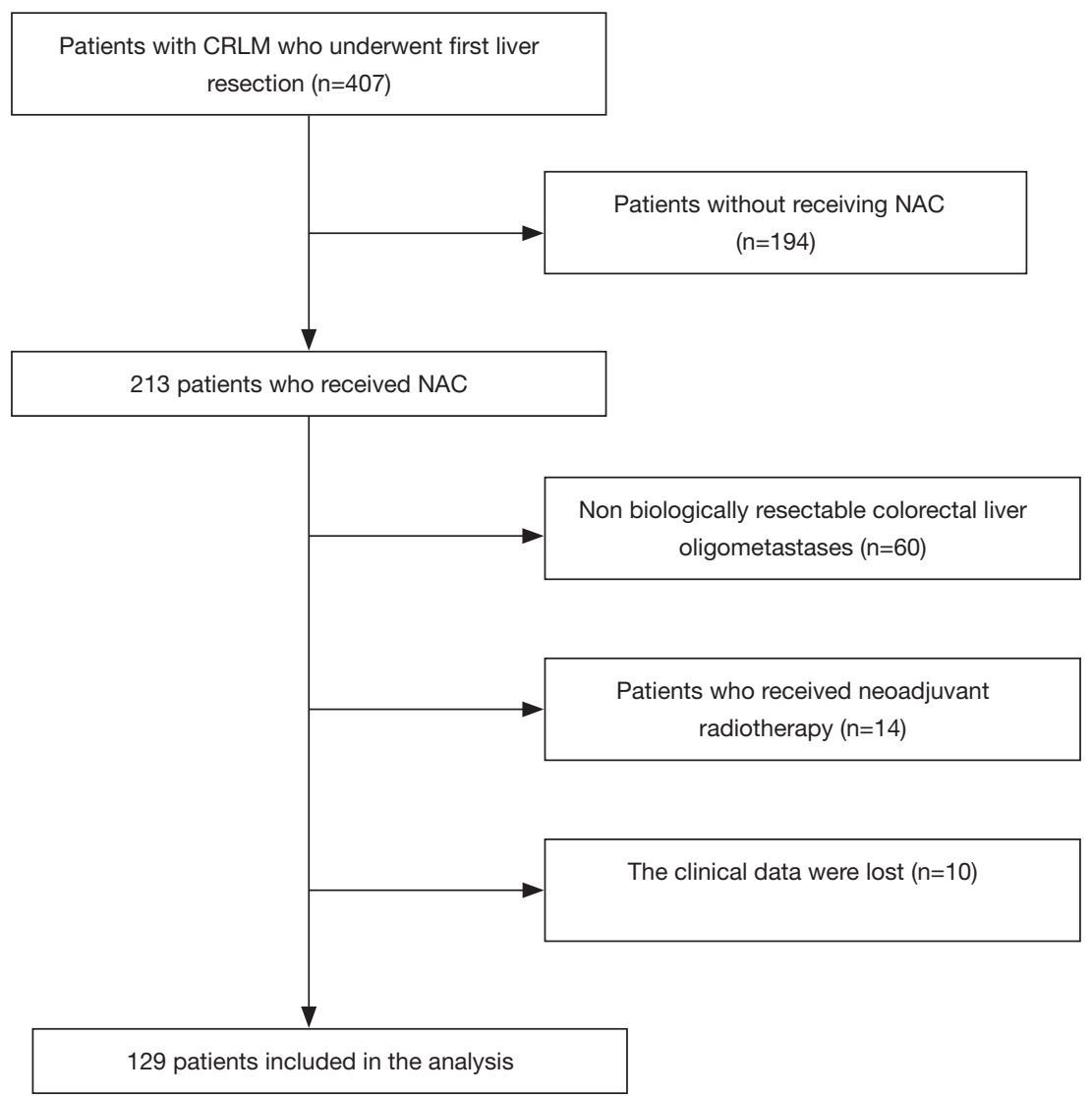

Figure 1 Flow diagram for the selection of CLOM in this study. CLOM, colorectal liver oligometastases; CRLM, colorectal cancer liver metastasis; NAC, neoadjuvant chemotherapy.

MDT may consider several factors including surgical difficulties, the efficacy of NAC, the NAC toxicities and the physical conditions of patients. The decision on the number of NAC cycles is a comprehensive consideration and varies for each patient. The MDT team will make the appropriate choice based on the characteristics of each patient. The postoperative chemotherapy regimens and the number of cycles depended on the NAC response, NAC cycles and tumour pathology. Tumour regression was graded as described by tumour regression grades (TRGs) (15). Tumour regression grades 1-3 were defined as favourable tumour responses, and grades 4-5 were defined as poor tumour responses. NAC toxicity was assessed according to the NCI-CTCAE (version 4.0).

$\mathrm{R} 0$ resection was defined as resected tumour lesions with a surgical margin of more than $1 \mathrm{~mm}$. Major liver resections were defined as resections of more than two liver segments. CRLM resection included simultaneous CRLM resection and heterochronous CRLM resection. Simultaneous resection was described as both primary and metastatic tumours resected at the same time. The postoperative complications were categorized by the Dindo-Clavien classification (16), with minor complications defined as grade I or II and major complications defined as grade III, $\mathrm{IV}$, or $\mathrm{V}$.

\section{Follow-up and outcome}

All patients were followed up 1 month after surgery, every 3 months thereafter. At each follow-up, CT or MRI scan, serum carcinoembryonic antigen (CEA) measurement and liver function test were routinely conducted. The tumor progression was evaluated by imaging and serum CEA measurement. After tumor progression, patients received surgery, radiofrequency ablation, transcatheter arterial chemoembolization, chemotherapy or targeted therapies, as appropriate. OS was defined as the time from surgery to death (all causes) or date of last follow-up. PFS was defined 
as the time from surgery to the first recorded evidence of progression, in cases without progression, to the last followup data or death.

\section{Statistical analysis}

Categorical variables are presented as frequencies with percentages, and continuous variables are presented as medians with interquartile ranges (IQRs). The perioperative clinicopathologic characteristics were compared using the $\chi^{2}$ and Mann-Whitney $\mathrm{U}$ tests, as appropriate. X-tile analysis (17) was implemented to investigate the optimal cut-off point for the number of NAC cycles. Propensity score matching was performed to reduce selection bias and quantify the possible associations between clinicopathologic characteristics and survival. To minimize the impact of other confounding factors on prognosis, we used a propensity score analysis to match these two types of patients (patients with NAC cycles $\leq 5$ and patients with NAC cycles $>5$ ) in a 1:1 ratio using the nearest neighbor matching. The confounders used for matching included major liver resection, multiple metastases, NAC toxicity and targeted therapy. This study used the Kaplan-Meier method to estimate the progression-free survival (PFS) and overall survival (OS) of all patients and statistically compared the data with log-rank tests. A forward LR Cox regression model was created to identify prognostic factors influencing OS and PFS, with results presented as hazard ratios (HRs) with $95 \%$ confidence intervals (95\% CI). Variables with $\mathrm{P}<0.10$ in univariable analysis were included in the multivariable analysis. All analyses were performed using SPSS, version 22 software (Armonk, NY, USA). $\mathrm{P}<0.05$ was considered significant.

\section{Results}

\section{Patients}

The detailed baseline characteristics of the included patients are shown in Table 1. A total of 129 patients were included, including 75 males and 54 females, with a median age of 56 years (IQR, 49-63, range, 28-79). Forty-seven patients (36.4\%) were aged older than 60 years. A BMI $>24 \mathrm{~kg} / \mathrm{m}^{2}$ was observed in 60 patients. The primary tumour site was the rectum in fifty-seven patients (44.2\%). Positive lymph nodes were found in more than half of the patients $(60.5 \%)$. The stage $\mathrm{pN} 1$ tumours were observed in 53 patients, and stage $\mathrm{pN} 2$ tumours were observed in 25 patients. Stage
pT3-pT4 tumours were observed in $83.7 \%$ of the patients. A single liver metastasis was present in 43 patients (33.3\%) $v s$. multiple metastases in 86 patients $(66.7 \%)$, with a median of 2 metastases (IQR, 1.0-3.0, range, 1-5). The maximum diameter of the largest liver lesion was $>3 \mathrm{~cm}$ in 61 patients, with a median diameter of $2.7 \mathrm{~cm}$ (IQR, 1.8-4.0); 87.6\% of the patients had synchronous liver metastases. A total of $44.2 \%$ of the patients had a bilobar distribution of liver lesions.

\section{Neoadjuvant chemotherapy and operative details}

The median number of NAC cycles was 4 (IQR, 3-6). An oxaliplatin-based regimen was administered for 95 patients $(73.6 \%)$, and targeted therapy was added in 41 cases $(31.8 \%)$. NAC toxicities were observed in 95 patients $(73.6 \%)$, including 53 patients with haematologic toxicities and 59 patients with gastrointestinal toxicity. A total of 52 patients had neutropenia, and 9 patients had liver toxicity. There was no mortality due to NAC toxicity. Fifty-two patients $(40.3 \%)$ had a favourable histological response (TRG 1-3). Seventy-four patients (57.4\%) were treated with postoperative chemotherapy. Major resection was performed in 61 patients $(47.3 \%)$, while minor liver resection was performed in 68 patients $(52.7 \%)$. Thirty-one patients underwent heterochronous resection. R0 resection was achieved in 93 patients (72.1\%).

\section{Clinicopathologic characteristics of the groups with a bigh number of $N A C$ cycles group and a low number of NAC cycles}

$\mathrm{X}$-tile software was used to determine that 5 was the optimal cut-off value for the number of NAC cycles (Figure 2). Based on the number of NAC cycles, 129 patients were classified into the group with a low number of NAC cycles ( $\leq 5$ cycles, $n=80$ ) and the group with a high number of NAC cycles $(>5$ cycles, $n=49$ ). In the group with a low number of NAC cycles, the median NAC cycles was 3.5 (IQR, 2.0-4.0). In the group with a high number of NAC cycles, the median NAC cycles was 7 (IQR, 6.0-8.5). The associations between NAC cycles and various clinicopathological features are shown in Table 1.

There were no significant differences in the R0 resection rate, pathological response, $\mathrm{pT}$ stage, presence of nodepositive primary tumour, preoperative CEA, metastasis diameter or bilobar distribution between the groups with a low number of NAC cycles group and a high number of 
Table 1 Patient and tumour characteristics

\begin{tabular}{|c|c|c|c|c|c|c|c|c|}
\hline Item & \multicolumn{4}{|c|}{ Before propensity matching } & \multicolumn{4}{|c|}{ After propensity matching } \\
\hline Male & $46(57.5)$ & $29(59.2)$ & 0.851 & $75(58.1)$ & $19(55.9)$ & $22(64.7)$ & 0.457 & $41(60.3)$ \\
\hline ASA score 3-4 & $9(11.3)$ & $7(14.32)$ & 0.612 & $16(12.4)$ & $3(8.8)$ & $5(14.7)$ & 0.452 & $8(11.8)$ \\
\hline $\begin{array}{l}\text { Preoperative CEA } \\
>10 \mathrm{ng} / \mathrm{mL}\end{array}$ & $33(41.3)$ & $19(38.8)$ & 0.781 & $52(40.3)$ & $16(47.1)$ & $14(41.2)$ & 0.625 & $30(44.1)$ \\
\hline Synchronous metastasis & $70(87.5)$ & $43(87.8)$ & 0.966 & $113(87.6)$ & $29(85.3)$ & $30(88.2)$ & 0.720 & $59(86.8)$ \\
\hline Major liver resection & $32(40.0)$ & $29(59.2)$ & 0.034 & $61(47.3)$ & $18(52.9)$ & $23(67.6)$ & 0.215 & $41(60.3)$ \\
\hline $\begin{array}{l}\text { Heterochronous } \\
\text { resection }\end{array}$ & $16(20.0)$ & $15(30.6)$ & 0.171 & $31(24.0)$ & $5(14.7)$ & $11(32.4)$ & 0.086 & $16(23.5)$ \\
\hline Bilobar distribution & $31(38.8)$ & $26(53.1)$ & 0.112 & $57(44.2)$ & $16(47.1)$ & $21(61.8)$ & 0.223 & $37(54.4)$ \\
\hline $\begin{array}{l}\text { Diameter of metastases } \\
>3 \mathrm{~cm}\end{array}$ & $34(42.5)$ & $27(55.1)$ & 0.164 & $61(47.3)$ & $17(50.0)$ & $21(61.8)$ & 0.329 & $38(55.9)$ \\
\hline Multiple metastases & $47(58.8)$ & $39(79.6)$ & 0.015 & $86(66.7)$ & $27(79.4)$ & $30(88.2)$ & 0.323 & $57(83.8)$ \\
\hline Poor differentiation & $18(22.5)$ & $11(22.4)$ & 0.995 & $29(22.5)$ & $9(26.5)$ & $9(26.5)$ & 1.000 & $18(26.5)$ \\
\hline \multicolumn{9}{|c|}{ Preoperative chemotherapy } \\
\hline $\begin{array}{l}\text { Oxaliplatin-based } \\
\text { regiment }\end{array}$ & $62(77.5)$ & $33(67.3)$ & 0.336 & $95(73.6)$ & $29(85.3)$ & $23(67.6)$ & 0.117 & $52(76.5)$ \\
\hline $\begin{array}{l}\text { Irinotecan-based } \\
\text { regiment }\end{array}$ & $9(11.3)$ & $6(12.2)$ & & $15(11.6)$ & $3(8.8)$ & $3(8.8)$ & & $6(8.8)$ \\
\hline Oxaliplatin + irinotecan & $9(11.3)$ & $10(20.5)$ & & $19(14.8)$ & $2(5.9)$ & $8(23.6)$ & & $10(14.7)$ \\
\hline Targeted therapy & $14(17.5)$ & $27(55.1)$ & $<0.001$ & $41(31.8)$ & $11(32.4)$ & $12(35.3)$ & 0.798 & $23(33.8)$ \\
\hline Pathological response & $30(37.5)$ & $22(44.9)$ & 0.406 & $52(40.3)$ & $16(47.1)$ & $12(35.3)$ & 0.324 & $28(41.2)$ \\
\hline $\begin{array}{l}\text { Post-operative } \\
\text { complications }\end{array}$ & $40(50.0)$ & $24(49.0)$ & 0.910 & $64(49.6)$ & $19(55.9)$ & $17(50.0)$ & 0.627 & $36(52.9)$ \\
\hline $\begin{array}{l}\text { Postoperative } \\
\text { chemotherapy }\end{array}$ & 49 (61.3) & 25 (51.0) & 0.254 & $74(57.4)$ & $22(64.7)$ & $19(55.9)$ & 0.457 & $41(60.3)$ \\
\hline
\end{tabular}

${ }^{a}$, KRAS status was available in 76 patients before propensity matching and in 44 patients after propensity matching. NAC, neoadjuvant chemotherapy; BMI, body mass index; ASA, American society of anesthesiologists physical status classification; CEA, carcinoembryonic antigen. 


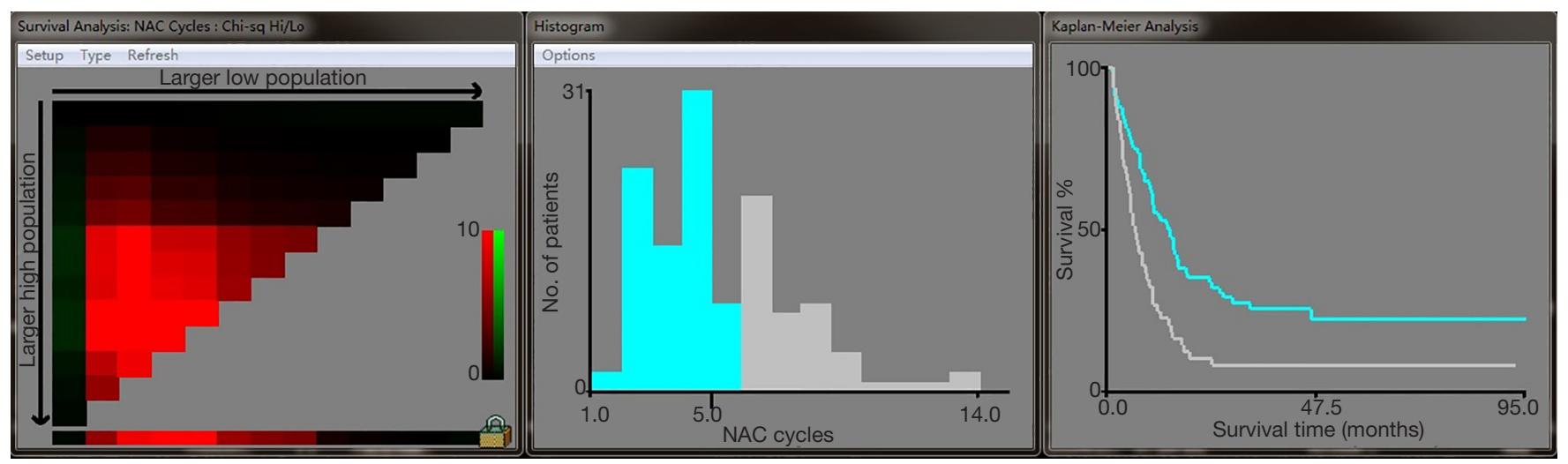

Figure $2 \mathrm{X}$-tile plots of the NAC cycles and the PFS of patients with CLOM who underwent curative resection. The optimal cut-off value of the NAC cycles was 5 . Histogram of the entire cohort divided into a low number of NAC cycles and a high number of NAC cycles subgroups according to the optimal cut-off value of 5. Blue bars represent the low NAC cycles group, and grey bars represent the high NAC cycles group. Kaplan-Meier plot of PFS in groups stratified using the optimal cut-off value of NAC cycles. Blue curves represent the low NAC cycles group, and grey curves represent the high NAC cycles group. NAC, neoadjuvant chemotherapy; PFS, progression-free survival.

NAC cycles. In contrast, compared to patients with a low number of NAC cycles, patients with a high number of NAC cycles were more likely to have NAC toxicity $(87.8 \%$ vs. $65.0 \%, \mathrm{P}=0.004)$, multiple liver metastases $(79.6 \%$ vs. $58.8 \%, \mathrm{P}=0.015)$ and major liver resection $(59.2 \%$ vs. $40.0 \%, \mathrm{P}=0.034)$.

To eliminate bias between the two groups, 1:1 propensity matching was conducted. The analysis of heterogeneity after propensity matching between 34 patients with a high number of NAC cycles and 34 patients with a low number of NAC cycles revealed an almost equal distribution of the following characteristics: age, ASA score, complications, NAC toxicity, number of liver metastases and surgery strategy (Table 1).

\section{Association between NAC cycles and postoperative complications}

No perioperative mortality occurred, and 64 patients (49.6\%) experienced complications (27 major complications; 37 minor complications). No liver failure was observed in this study. In the group with a low number of NAC cycles, 40 patients developed complications (17 major complications and 23 minor complications). In the group with a high number of NAC cycles, 24 patients developed complications (10 major complications and 14 minor complications). We further investigated the complication rates to address the question of whether patients with a high number of NAC cycles suffered from a higher incidence of postoperative complications. No difference in postoperative complications $(\mathrm{P}=0.910)$, minor complications $(\mathrm{P}=0.983)$ or major complications $(\mathrm{P}=0.909)$ was observed between the two groups.

\section{Association between the number of NAC cycles and survival}

\section{Before 1:1 propensity matching}

The median follow-up time was 46 months. One hundred and three patients (79.84\%) experienced disease recurrence, and 65 patients $(50.39 \%)$ died. The median OS was 42.3 months (95\% CI: 34.3-50.3), and the median PFS was 9.9 months (95\% CI: 8.2-11.6). Compared to patients with a high number of NAC cycles, the patients with a low number of NAC cycles had better a PFS $(\mathrm{P}<0.001$, mPFS: 13.3 vs. 6.0 months $)$ and better $\mathrm{OS}(\mathrm{P}=0.008, \mathrm{mOS}: 57.7$ vs. 32.1 months) (Figure 3).

Univariate analysis revealed that age $\leq 60$ years, non-R0 resection, major liver resection, bilobar distribution, multiple metastases, node-positive primary tumour, $>5$ NAC cycles and targeted therapy were associated with a reduced PFS. Multivariate analysis showed that $>5 \mathrm{NAC}$ cycles $(\mathrm{HR}=1.808,95 \% \mathrm{CI}: 1.205-2.712, \mathrm{P}=0.004)$ and node-positive primary tumours $(\mathrm{HR}=1.858,95 \% \mathrm{CI}$ : 1.204-2.868, $\mathrm{P}=0.005)$ were independently associated with a reduced PFS and $\mathrm{R} 0$ resection $(\mathrm{HR}=0.642,95 \% \mathrm{CI}$ : 
A

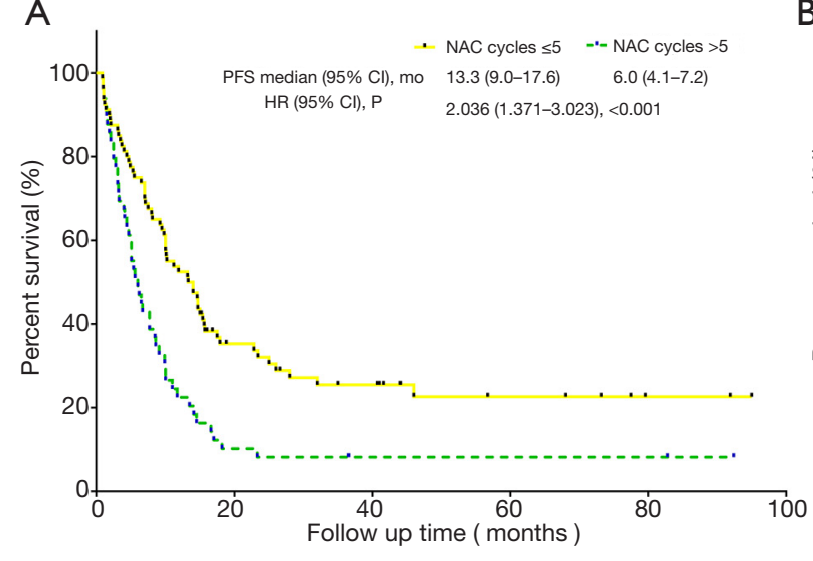

B

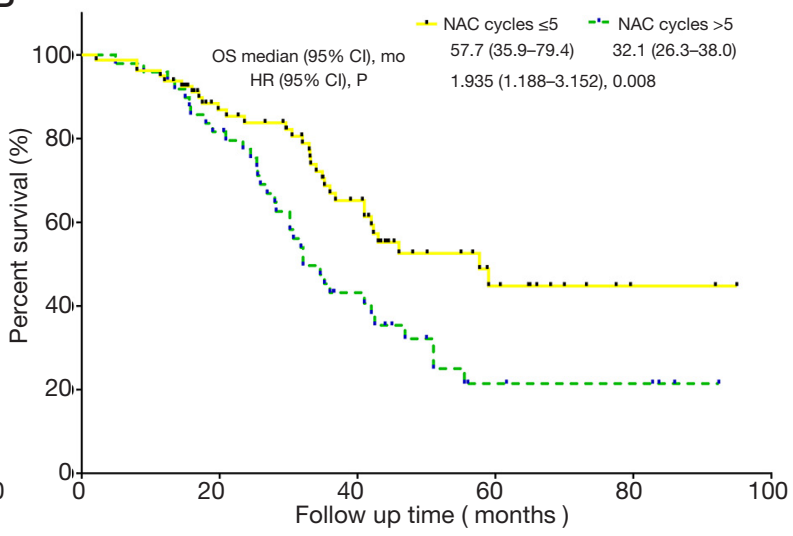

Figure 3 Survival analysis before propensity matching. (A) PFS analysis, (B) OS analysis. NAC, neoadjuvant chemotherapy; PFS, progression-free survival; OS, overall survival.

0.419-0.982, $\mathrm{P}=0.041)$ was an independent predictor of a prolonged PFS (Table 2).

Univariate analysis revealed that major liver resection, complications and $>5 \mathrm{NAC}$ cycles were associated with a reduced OS, and postoperative chemotherapy was associated with a prolonged OS. Multivariate analysis showed that bilobar distribution (HR $=1.744$, 95\% CI: 1.039-2.928, $\mathrm{P}=0.035$ ), complications (HR $=2.207,95 \%$ CI: $1.321-3.686$, $\mathrm{P}=0.002)$ and $>5$ NAC cycles (HR $=1.723$, 95\% CI: $1.041-$ 2.851, $\mathrm{P}=0.034$ ) were independent predictors of a reduced OS and postoperative chemotherapy ( $\mathrm{HR}=0.557,95 \%$ CI: $0.336-0.924, \mathrm{P}=0.024)$ was an independent predictor of a prolonged OS (Table 2).

\section{Subgroup analysis of different $\mathrm{NAC}$ regimens}

Ninety-five patients received an oxaliplatin-based regimen. In the oxaliplatin-based regimen group, patients with a low number of NAC cycles had better PFS $(\mathrm{P}<0.001$, mPFS: 14.7 vs. 5.4 months) and better OS ( $\mathrm{P}=0.018$, mOS: 57.7 vs. 41.0 months) than those with a higher number of NAC cycles (Figure 4). The number of patients receiving irinotecan-based regimens (15 cases) or oxaliplatin + irinotecan regimens (19 cases) was limited. Thus, a survival analysis could not be conducted.

\section{After 1:1 propensity matching}

Compared to patients with a high number of NAC cycles, the patients with a low number of NAC cycles had significantly better PFS $(\mathrm{P}<0.001, \mathrm{mPFS}: 14.7$ vs. 5.6 months $)$ and better $\mathrm{OS}(\mathrm{P}=0.012$, mOS: 59.0 vs. 31.8 months) (Figure 5).

Univariate analysis revealed that ASA score 3-4, non-R0 resection, major liver resection, bilobar distribution, multiple metastases, node-positive primary tumour, $>5$ NAC cycles and targeted therapy were associated with a reduced PFS and postoperative chemotherapy was associated with a prolonged PFS. Multivariate analysis showed that bilobar distribution ( $\mathrm{HR}=2.176,95 \% \mathrm{CI}: 1.203-3.935, \mathrm{P}=0.010)$ and $>5$ NAC cycles $(\mathrm{HR}=2.265,95 \%$ CI: $1.281-4.007$, $\mathrm{P}=0.005)$ were independent predictors of a reduced PFS and postoperative chemotherapy ( $\mathrm{HR}=0.517,95 \% \mathrm{CI}$ : $0.291-0.921, \mathrm{P}=0.025)$ was an independent predictor of a prolonged PFS (Table 3).

Univariate analysis revealed that non-R0 resection, metastasis diameter $\geq 3 \mathrm{~cm}$, node-positive primary tumour, NAC toxicity and $>5$ NAC cycles were associated with a reduced OS and postoperative chemotherapy was associated with a prolonged OS. Multivariate analysis showed that $>5$ NAC cycles (HR $=2.813,95 \%$ CI: $1.359-5.822$, $\mathrm{P}=0.005)$ was an independent predictor of a reduced OS and postoperative chemotherapy ( $\mathrm{HR}=0.312,95 \% \mathrm{CI}$ : 0.153-0.636, $\mathrm{P}=0.001$ ) was an independent predictor of a prolonged OS (Table 3). The result that $>5$ NAC cycles was an independent predictor of a reduced PFS and reduced OS was consistent with the result before propensity matching.

\section{Subgroup analysis in different NAC regiments}

Fifty-two patients received oxaliplatin-based regimen. In the oxaliplatin-based regimen group, patients with a low number of NAC cycles had better PFS $(\mathrm{P}<0.001, \mathrm{mPFS}$ : 17.5 vs. 5.6 months $)$ and better OS $(\mathrm{P}=0.008$, mOS: 59.0 vs. 31.8 months) (Figure 6). The number of patients receiving irinotecan-based regimen (6 cases) or oxaliplatin + irinotecan regimen (10 cases) was limited. Thus, a survival 
Table 2 Univariate and multivariate analyses of predictive factors of PFS and OS for CLOM patients before propensity matching

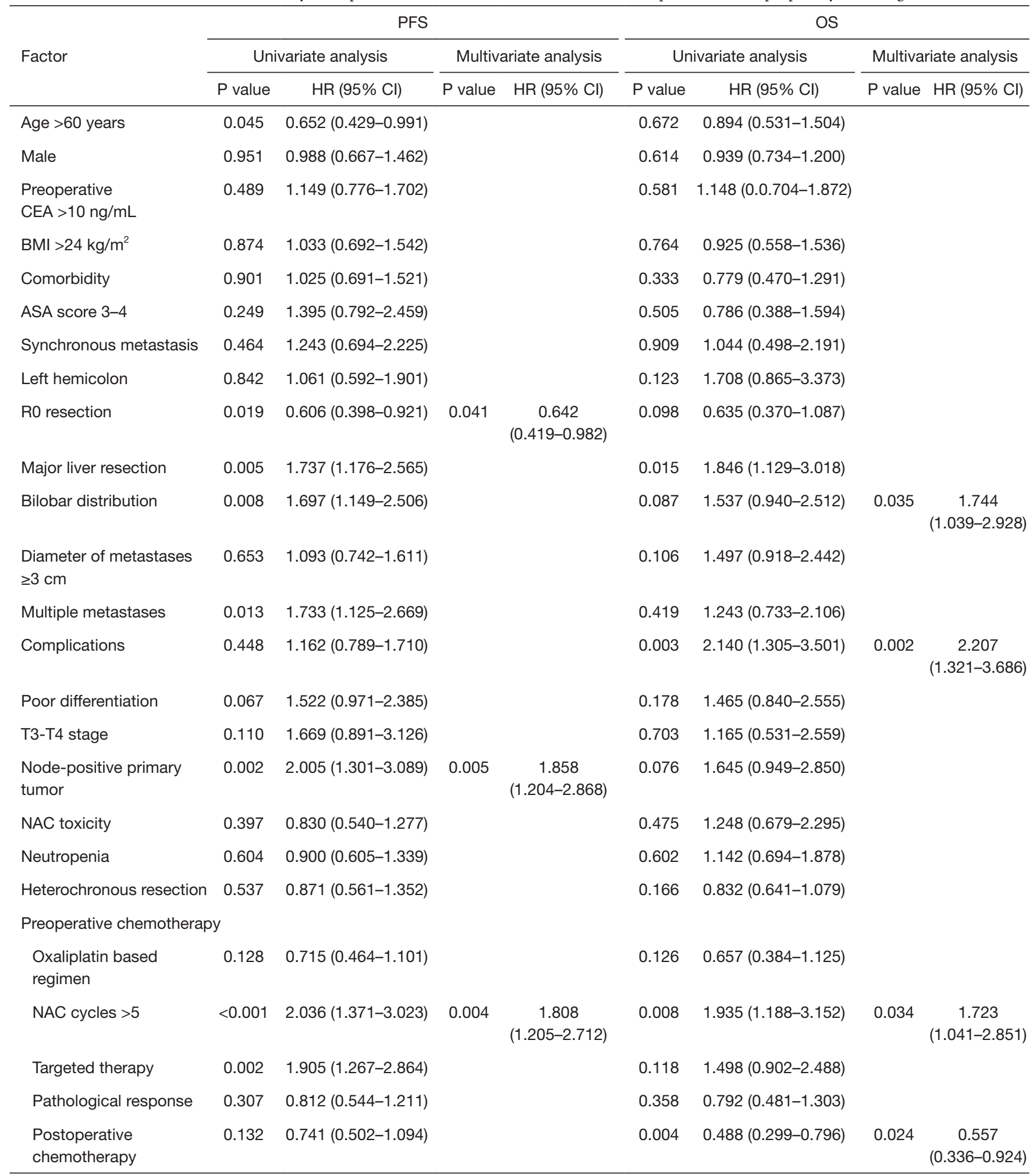

PFS, progression-free survival; OS, overall survival; CLOM, colorectal liver oligometastases; NAC, neoadjuvant chemotherapy; CEA, carcinoembryonic antigen; BMI, body mass index; ASA, American society of anesthesiologists physical status classification. 
A

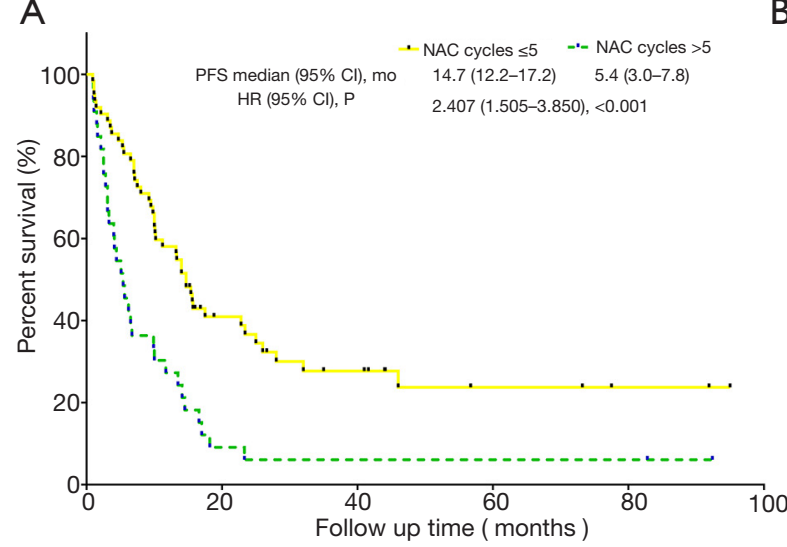

B

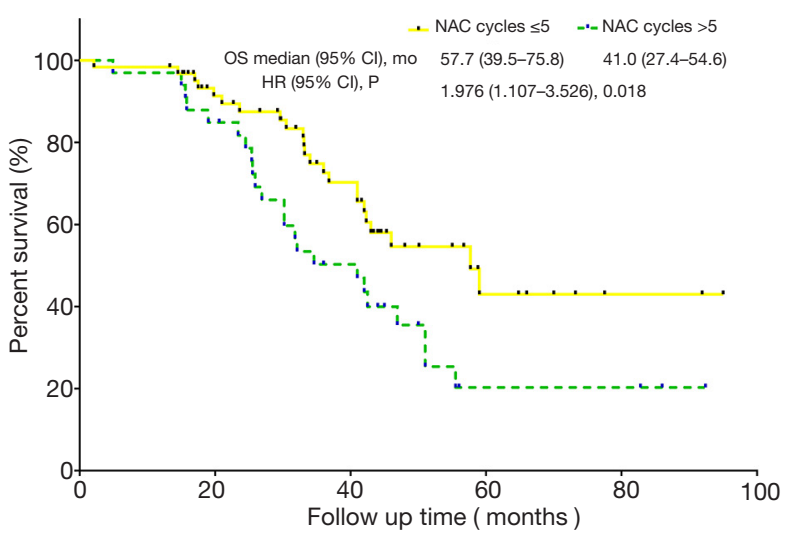

Figure 4 Survival analysis in patients receiving oxaliplatin-based regimen before propensity matching. (A) PFS analysis, (B) OS analysis. NAC, neoadjuvant chemotherapy; PFS, progression-free survival; OS, overall survival.

A

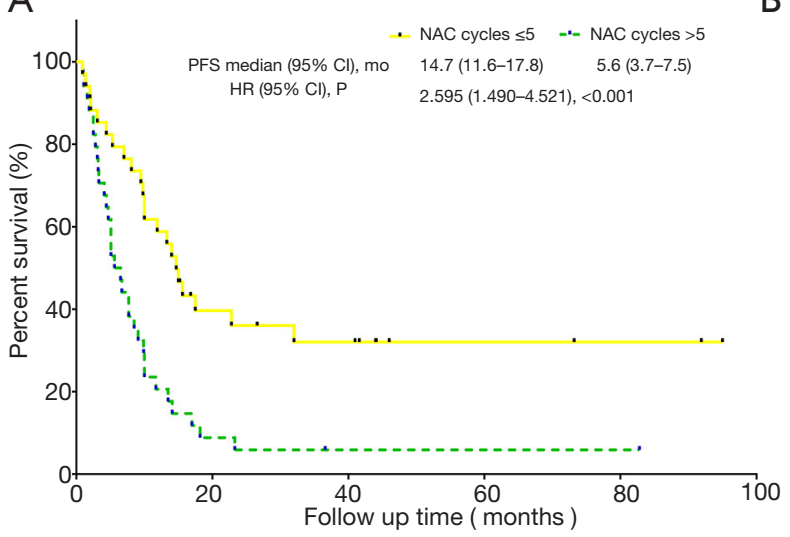

B

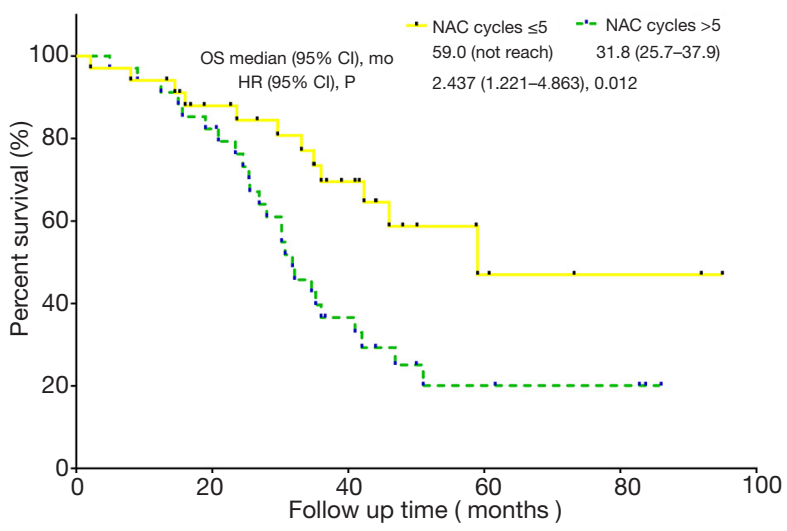

Figure 5 Survival analysis after propensity matching. (A) PFS analysis, (B) OS analysis. NAC, neoadjuvant chemotherapy; PFS, progressionfree survival; OS, overall survival.

analysis could not be conducted.

\section{Discussion}

To the best of our knowledge, this study was the first to investigate the optimal number of NAC cycles for biologically resectable CLOM. X-tile analysis was used and determined that 5 was the optimal cut-off value for NAC cycles. The results of this study revealed that CLOM patients receiving $\leq 5$ NAC cycles had favourable PFS and OS in the multivariate analysis. In a separate analysis of patients receiving an oxaliplatin-based regimen, the study yielded consistent results. A 1:1 propensity score matching analysis confirmed this finding. Thus, 5 NAC cycles represent an inflection point for resection with unfavourable outcomes.

For CRLM patients, the total number of liver metastases is an important factor for the choice of treatment strategies. Patients with more than five liver metastases were considered to have biological unresectable disease and were recommended to receive NAC (18). Patients with $\leq 5$ CRLM, especially those without extrahepatic metastases, were regarded as a unique subgroup (CLOM) $(6,7)$. CLOM is a relatively early stage of biological invasion between localized primary tumours and widespread metastatic tumours. Effective control of tumour progression at this stage can significantly improve the prognosis of patients. For biologically resectable CLOM, NAC is increasingly recommended because of its several potential advantages, including early elimination of distant micrometastases, 
Table 3 Univariate and multivariate analyses of predictive factors of PFS and OS for CLOM patients after propensity matching

\begin{tabular}{|c|c|c|c|c|c|c|c|c|}
\hline \multirow{3}{*}{ Factor } & \multicolumn{4}{|c|}{ PFS } & \multicolumn{4}{|c|}{ OS } \\
\hline & \multicolumn{2}{|c|}{ Univariate analysis } & \multicolumn{2}{|c|}{ Multivariate analysis } & \multicolumn{2}{|c|}{ Univariate analysis } & \multicolumn{2}{|c|}{ Multivariate analysis } \\
\hline & $P$ value & $\mathrm{HR}(95 \% \mathrm{Cl})$ & $P$ value & HR (95\% Cl) & $P$ value & HR $(95 \% \mathrm{Cl})$ & $P$ value & HR (95\%Cl) \\
\hline Age $>60$ years & 0.319 & $0.742(0.413-1.334)$ & & & 0.640 & $1.175(0.597-2.315)$ & & \\
\hline Male & 0.799 & $1.074(0.621-1.858)$ & & & 0.885 & $0.976(0.699-1.362)$ & & \\
\hline $\mathrm{BMI}>24 \mathrm{~kg} / \mathrm{m}^{2}$ & 0.534 & $0.838(0.478-1.469)$ & & & 0.591 & $1.203(0.613-2.362)$ & & \\
\hline Comorbidity & 0.857 & $1.052(0.605-1.831)$ & & & 0.769 & $0.904(0.460-1.777)$ & & \\
\hline ASA score 3-4 & 0.045 & 2.207 (1.016-4.792) & & & 0.254 & $1.617(0.708-3.690)$ & & \\
\hline Major liver resection & 0.016 & $2.032(1.141-3.619)$ & & & 0.079 & $1.862(0.930-3.729)$ & & \\
\hline Bilobar distribution & 0.002 & $2.519(1.424-4.458)$ & 0.010 & $\begin{array}{c}2.176 \\
(1.203-3.935)\end{array}$ & 0.085 & $1.825(0.921-3.616)$ & & \\
\hline $\begin{array}{l}\text { Diameter of metastases } \\
\geq 3 \mathrm{~cm}\end{array}$ & 0.973 & 1.009 (0.588-1.732) & & & 0.030 & $2.164(1.078-4.345)$ & & \\
\hline Multiple metastases & 0.030 & $2.792(1.104-7.063)$ & & & 0.529 & $1.396(0.494-3.947)$ & & \\
\hline Complications & 0.221 & $0.714(0.416-1.224)$ & & & 0.488 & $1.257(0.658-2.404)$ & & \\
\hline Poor differentiation & 0.174 & $1.511(0.834-2.740)$ & & & 0.084 & $1.865(0.919-3.782)$ & & \\
\hline $\begin{array}{l}\text { Heterochronous } \\
\text { resection }\end{array}$ & 0.304 & $0.730(0.401-1.330)$ & & & 0.704 & $0.932(0.647-1.341)$ & & \\
\hline \multicolumn{9}{|l|}{ Preoperative chemotherapy } \\
\hline $\begin{array}{l}\text { Oxaliplatin based } \\
\text { regimen }\end{array}$ & 0.037 & $0.521(0.282-0.960)$ & & & 0.256 & $0.663(0.327-1.347)$ & & \\
\hline NAC cycles $>5$ & 0.001 & $2.595(1.490-4.521)$ & 0.005 & $\begin{array}{c}2.265 \\
(1.281-4.007)\end{array}$ & 0.012 & $2.437(1.221-4.863)$ & 0.005 & $\begin{array}{c}2.813 \\
(1.359-5.822)\end{array}$ \\
\hline Targeted therapy & 0.035 & $1.824(1.044-3.185)$ & & & 0.375 & $1.362(0.688-2.700)$ & & \\
\hline Pathological response & 0.393 & $0.785(0.451-1.367)$ & & & 0.257 & $0.676(0.343-1.331)$ & & \\
\hline $\begin{array}{l}\text { Postoperative } \\
\text { chemotherapy }\end{array}$ & 0.007 & $0.470(0.271-0.813)$ & 0.025 & $\begin{array}{c}0.517 \\
(0.291-0.921)\end{array}$ & 0.001 & $0.320(0.165-0.621)$ & 0.001 & $\begin{array}{c}0.312 \\
(0.153-0.636)\end{array}$ \\
\hline
\end{tabular}

PFS, progression-free survival; OS, overall survival; CLOM, colorectal liver oligometastases; NAC, neoadjuvant chemotherapy; CEA, carcinoembryonic antigen; BMI, body mass index; ASA, American society of anesthesiologists physical status classification. 
A

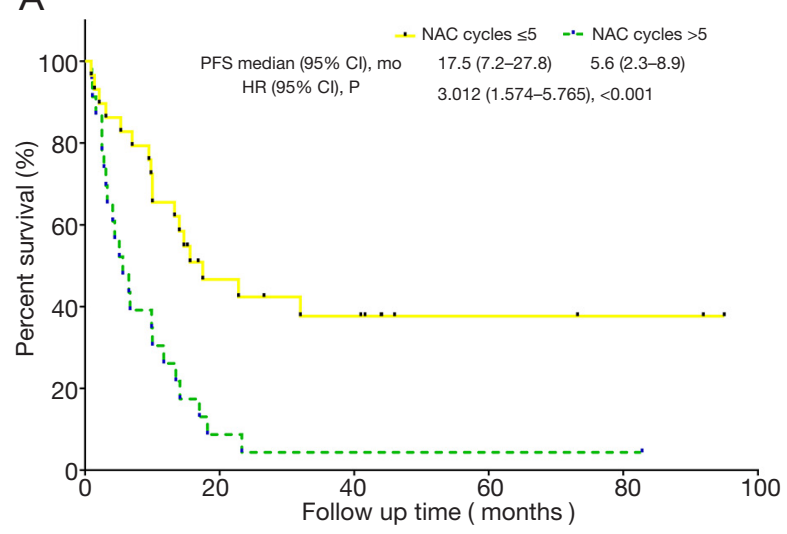

B

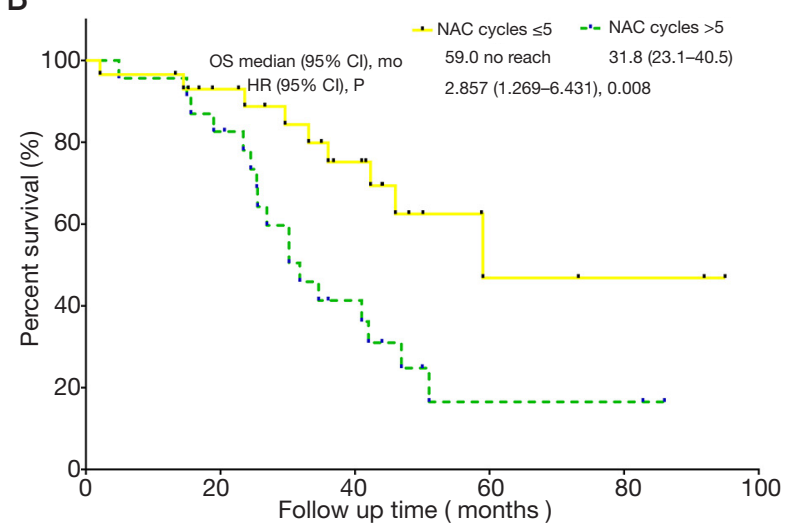

Figure 6 Survival analysis in patients receiving oxaliplatin-based regimen after propensity matching. (A) PFS analysis; (B) OS analysis. NAC, neoadjuvant chemotherapy; PFS, progression-free survival; OS, overall survival.

improved resectability that leads to survival benefits, and in vivo evaluation of chemotherapy regimen response (8). However, clinicians need to address an important question: what is the optimal number of NAC cycles for CLOM patients? For unresectable CRLM, the choice of the number of NAC cycles was determined to achieve resectability. Unlike that for unresectable CRLM, the number of NAC cycles for biologically resectable CLOM can be chosen objectively. However, an insufficient number of cycles of NAC cannot lead to the maximum benefits and realize the advantages of NAC. In contrast, an excessive number of NAC cycles may cause adverse effects such as impaired immune function, reduced sensitivity to chemotherapy regimens and an elevated risk of developing distant metastasis. It is necessary for us to investigate the optimal number of NAC cycles.

There is still no consensus on the number of NAC cycles for biologically resectable CLOM patients. The NCCN guidelines (11) recommend the option of NAC for resectable CRLM for a period of 2-3 months but do not specify how many cycles of NAC would benefit patients the most. This study implemented X-tile analyses to objectively identify the optimal number of NAC cycles for CLOM instead of arbitrarily determining the cut-off point of NAC cycles. Five NAC cycles for CLOM was the inflection point in survival, and $>5 \mathrm{NAC}$ cycles led to unfavourable survival. The mechanisms of this result may be as follows: (I) more NAC cycles would impair immune function and nutritional status $(19,20)$, which is not conducive to the prognosis of patients (21). Some studies have shown that prolonging chemotherapy cycles leads to the occurrence of sarcopenia (22) and chemotherapy toxicities (12), and sarcopenia and NAC chemotherapy toxicities are significantly related to poor patient prognosis $(13,23)$. (II) The sensitivity of the tumour to the chemotherapy regimen may decrease as the chemotherapy cycles are extended (24). Once chemotherapy regimens cannot completely control the tumour, the risk of local progression or metastasis increases and CRLM can even convert into unresectable liver metastasis. In addition, the reduction in tumour sensitivity to chemotherapy regimens would not be conducive to postoperative adjuvant chemotherapy and palliative chemotherapy after recurrence. (III) Prolonged chemotherapy cycles may lead to further reductions in the size of liver metastasis. There is the possibility that local metastatic tumours disappear and cannot be found by imaging (25); thus, these metastases cannot be completely removed by surgery. Some studies have shown that more than half of these metastases will recur in situ (26,27). (IV) A few preclinical studies have demonstrated that NAC may induce cancer metastasis through a tumour microenvironment of metastasis-mediated and/or extracellular vesicle-mediated mechanisms $(28,29)$.

When investigating the optimal number of NAC cycles, the study has to consider the heterogenicity of NAC regimens. To address this bias, this study used a 1:1 propensity score matching analysis to balance the NAC regimen differences between the two groups. In addition, we conducted subgroup analyses according to different NAC regimens. Although the study did not conduct subgroup analyses according to all NAC regimens because of the limited sample size (oxaliplatin-based regimen: 95; 
irinotecan-based regimen: 15 cases; oxaliplatin + irinotecan regimen: 19 cases), for patients receiving oxaliplatin-based regimen, the study yielded consistent results (patients who received $\leq 5 \mathrm{NAC}$ cycles had favourable PFS and OS) before and after propensity score matching analysis.

It is important to note that our study revealed that additional NAC cycles not only did not change the R0 resection rate and pathological response rate but also increased the risk of NAC toxicity. In addition, additional NAC cycles may increase the risk of perioperative bleeding due to increased tissue fragility and inflammatory response (30). In conclusion, we do not recommend NAC for more than five cycles.

There are several limitations in this study. First, this was a retrospective, single-institution study with a small sample size. Selection bias was inevitable and may have influenced our results. Second, the number of NAC cycles was determined by the MDT team, which had bias for outcome. In order to decrease the selection bias, the propensity score matching was performed in this study. There is no doubt that propensity score matching can decrease the selection bias but does not eliminate it and decrease the numbers eligible for analyses, which was the limitation of this study. Third, there is still a lack of high-level clinical evidence on whether CRLM patients should receive postoperative chemotherapy and on the optimal cycles of postoperative chemotherapy. The differences of postoperative chemotherapy in this study lead to the survival bias. Last, this study did not include the KRAS status in the analysis because only 76 patients had available KRAS statuses. Despite these limitations, we believe that our study results may provide valuable applicable information to routine clinical practice.

In conclusion, our retrospective analysis showed that fewer than 5 NAC cycles was optimal for biologically resectable CLOM patients. Giving more than 5 cycles of NAC was unnecessary because a higher number of NAC cycles leads to more unfavourable survival and increased NAC toxicity while leading to similar R0 resection rates and pathological response rates. Our findings should be confirmed by prospective and controlled trials.

\section{Acknowledgments}

Funding: This study was supported by the State Key Project on Infection Diseases of China (Grant No. 2017ZX10201021-007-003), the National The capital health research and development of special (2018-1-
4021), the National Natural Science Foundation of China (81672461, 81972311), CAMS Innovation Fund for Medical Sciences (CIFMS) (Grant no.2017-12M-4-002), the Non-profit Central Research Institution Fund of Chinese Academy of Medical Sciences (2019PT310026) and Sanming Project of Medicine in Shenzhen (No. SZSM202011010).

\section{Footnote}

Reporting Checklist: The authors have completed the STROBE reporting checklist. Available at http://dx.doi. org/10.21037/atm-20-4289

Data Sharing Statement: Available at http://dx.doi. org/10.21037/atm-20-4289

Conflicts of Interest: All authors have completed the ICMJE uniform disclosure form (available at http://dx.doi. org/10.21037/atm-20-4289). The authors have no conflicts of interest to declare.

Ethics Statement: The authors are accountable for all aspects of the work in ensuring that questions related to the accuracy or integrity of any part of the work are appropriately investigated and resolved. All participants gave informed consent before taking part in this study. This study was conducted with approval from the Institute Research Ethics Committee of the Cancer Hospital, Chinese Academy of Medical Sciences (ID:NCC2019C-016). The study conformed to the provisions of the Declaration of Helsinki (as revised in 2013).

Open Access Statement: This is an Open Access article distributed in accordance with the Creative Commons Attribution-NonCommercial-NoDerivs 4.0 International License (CC BY-NC-ND 4.0), which permits the noncommercial replication and distribution of the article with the strict proviso that no changes or edits are made and the original work is properly cited (including links to both the formal publication through the relevant DOI and the license). See: https://creativecommons.org/licenses/by-nc-nd/4.0/.

\section{References}

1. Bray F, Ferlay J, Soerjomataram I, Siegel RL, et al. Global cancer statistics 2018: GLOBOCAN estimates of incidence and mortality worldwide for 36 cancers in 185 
countries. CA Cancer J Clin 2018;68:394-424.

2. Engstrand J, Nilsson H, Stromberg C, et al. Colorectal cancer liver metastases - a population-based study on incidence, management and survival. BMC Cancer 2018; $18: 78$.

3. Fiorentini G, Sarti D, Aliberti C, et al. Multidisciplinary approach of colorectal cancer liver metastases. World J Clin Oncol 2017;8:190-202.

4. Chen Q, Zhao H, Wu J, et al. Preoperative D-dimer and Gamma-Glutamyltranspeptidase Predict Major Complications and Survival in Colorectal Liver Metastases Patients After Resection. Transl Oncol 2019;12:996-1004.

5. Van Cutsem E, Cervantes A, Adam R, et al. ESMO consensus guidelines for the management of patients with metastatic colorectal cancer. Ann Oncol 2016;27:1386-422.

6. Lin J, Peng J, Zhao Y, et al. Early recurrence in patients undergoing curative resection of colorectal liver oligometastases: identification of its clinical characteristics, risk factors, and prognosis. J Cancer Res Clin Oncol 2018;144:359-69.

7. Lu Z, Peng J, Wang Z, et al. High preoperative serum CA19-9 level is predictive of poor prognosis for patients with colorectal liver oligometastases undergoing hepatic resection. Med Oncol 2016;33:121.

8. Symonds LK, Cohen S(A) Use of perioperative chemotherapy in colorectal cancer metastatic to the liver. Gastroenterol Rep (Oxf) 2019;7:301-11.

9. Ribeiro HS, Costa WL, Jr, Diniz AL, et al. Extended preoperative chemotherapy, extent of liver resection and blood transfusion are predictive factors of liver failure following resection of colorectal liver metastasis. Eur J Surg Oncol 2013;39:380-5.

10. Kishi Y, Zorzi D, Contreras CM, et al. Extended preoperative chemotherapy does not improve pathologic response and increases postoperative liver insufficiency after hepatic resection for colorectal liver metastases. Ann Surg Oncol 2010;17:2870-6.

11. Network NCC. NCCN clinical practice guidelines in Oncology: Rectal Cancer (2019.V2). 2019.

12. Li J, Li Y, Zhong M, et al. Short-Course Versus Long-Course Chemoradiotherapy for Stage IE-IIE Extranodal Natural Killer/T cell Lymphoma, Nasal Type: A Multicenter Retrospective Study. Med Sci Monit 2018;24:2683-92.

13. Chen Q, Wu C, Zhao H, et al. Neo-adjuvant Chemotherapy-Induced Neutropenia Is Associated with Histological Responses and Outcomes after the Resection of Colorectal Liver Metastases. J Gastrointest Surg 2020;24:659-70.

14. Chen Q, Li C, Yang H, et al. Resection Combined with TKI Therapy for Resectable Liver Metastases of Gastrointestinal Stromal Tumours: Results from Three National Centres in China. J Gastrointest Surg 2020;24:1330-41.

15. Rubbia-Brandt L, Giostra E, Brezault C, et al. Importance of histological tumor response assessment in predicting the outcome in patients with colorectal liver metastases treated with neo-adjuvant chemotherapy followed by liver surgery. Ann Oncol 2007;18:299-304.

16. Dindo D, Demartines N, Clavien $\mathrm{P}(\mathrm{A})$ Classification of surgical complications: a new proposal with evaluation in a cohort of 6336 patients and results of a survey. Ann Surg 2004;240:205-13.

17. Camp RL, Dolled-Filhart M, Rimm DL. X-tile: a new bio-informatics tool for biomarker assessment and outcome-based cut-point optimization. Clin Cancer Res 2004;10:7252-9.

18. Diagnosis And Treatment Guidelines For Colorectal Cancer Working Group CS. Chinese Society of Clinical Oncology (CSCO) diagnosis and treatment guidelines for colorectal cancer 2018 (English version). Chin J Cancer Res 2019;31:117-34.

19. Wang WH, Xu HY, Zhao ZM, et al. Dynamic and significant changes of $T$-cell subgroups in breast cancer patients during surgery and chemotherapy. Int Immunopharmacol 2018;65:279-83.

20. Wu Y, Deng Z, Wang H, et al. Repeated cycles of 5-fluorouracil chemotherapy impaired anti-tumor functions of cytotoxic T cells in a CT26 tumor-bearing mouse model. BMC Immunol 2016;17:29.

21. Barret M, Malka D, Aparicio T, et al. Nutritional status affects treatment tolerability and survival in metastatic colorectal cancer patients: results of an AGEO prospective multicenter study. Oncology 2011;81:395-402.

22. Kurk SA, Peeters PHM, Dorresteijn B, et al. Impact of different palliative systemic treatments on skeletal muscle mass in metastatic colorectal cancer patients. J Cachexia Sarcopenia Muscle 2018;9:909-19.

23. Deng CY, Lin YC, Wu JS, et al. Progressive Sarcopenia in Patients With Colorectal Cancer Predicts Survival. AJR Am J Roentgenol 2018;210:526-32.

24. Mansoori B, Mohammadi A, Davudian S, et al. The Different Mechanisms of Cancer Drug Resistance: A Brief Review. Adv Pharm Bull 2017;7:339-48. 


\section{Page 14 of 14}

25. Tsilimigras DI, Ntanasis-Stathopoulos I, Paredes AZ, et al. Disappearing liver metastases: A systematic review of the current evidence. Surg Oncol 2019;29:7-13.

26. Kuhlmann K, van Hilst J, Fisher S, et al. Management of disappearing colorectal liver metastases. Eur J Surg Oncol 2016;42:1798-805.

27. Bischof DA, Clary BM, Maithel SK, et al. Surgical management of disappearing colorectal liver metastases. $\mathrm{Br}$ J Surg 2013;100:1414-20.

28. Keklikoglou I, Cianciaruso C, Guc E, et al. Chemotherapy

Cite this article as: Chen Q, Li X, Zhao J, Bi X, Li Z, Huang Z, Zhang Y, Zhou J, Zhao H, Cai J. What is the optimal number of neoadjuvant chemotherapy cycles for resectable colorectal liver oligometastases? Ann Transl Med 2021;9(1):7. doi: 10.21037/atm-20-4289

\section{Chen et al. The optimal number of NAC cycles for CLOM}

elicits pro-metastatic extracellular vesicles in breast cancer models. Nat Cell Biol 2019;21:190-202.

29. Karagiannis GS, Pastoriza JM, Wang Y, et al. Neoadjuvant chemotherapy induces breast cancer metastasis through a TMEM-mediated mechanism. Sci Transl Med 2017;9:eaan0026.

30. Hirokawa F, Asakuma M, Komeda K, et al. Is neoadjuvant chemotherapy appropriate for patients with resectable liver metastases from colorectal cancer? Surg Today 2019;49:82-9. 